\title{
In-depth analysis of traffic congestion using computational fluid dynamics (CFD) modeling method
}

\author{
Dazhi SUN ${ }^{1 *}$, Jinpeng $\mathrm{LV}^{1}$, S. Travis WALLER ${ }^{2}$ \\ 1. Department of Civil \& Architectural Engineering, Texas A\&M University-Kingsville, Texas 78363, USA \\ 2. Department of Civil Engineering, The University of Texas at Austin, Austin, Texas 78712, USA
}

\begin{abstract}
This paper introduces computational fluid dynamics (CFD), a numerical approach widely and successfully used in aerospace engineering, to deal with surface traffic flow related problems. After a brief introduction of the physical and mathematical foundations of CFD, this paper develops CFD implementation methodology for modeling traffic problems such as queue/platoon distribution, shockwave propagation, and prediction of system performance. Some theoretical and practical applications are discussed in this paper to illustrate the implementation methodology. It is found that CFD approach can facilitate a superior insight into the formation and propagation of congestion, thereby supporting more effective methods to alleviate congestion. In addition, CFD approach is found capable of assessing freeway system performance using less ITS detectors, and enhancing the coverage and reliability of a traffic detection system.
\end{abstract}

Key words: CFD; Euler's equation; shockwaves; queue/platoon; system performance monitoring

(C) 2011 JMT. All rights reserved.

\section{Introduction}

$\mathrm{I}$ $t$ has been over a half century since engineers and experts incorporated the theory of fluid dynamics in to transportation study. It began in the 1950's when Lighthill and Whitham [1] introduced a one-dimensional method, which allowed for the study of transportation problems using fluid dynamic method. Later, Richards [2] developed a simple traffic flow under the precondition that the movement of a group of discrete vehicles could be treated as a continuous flow and and the equation of the conservation of matter was given as

$$
\frac{\partial d}{\partial t}+\frac{\partial d \cdot v}{\partial x}=0
$$

which is also called the one-dimensional Euler's equation in fluid mechanics.

Since these early pioneering works, the study and use of the one-dimensional Euler's equation in traffic flow theory has continued to be a topic of interest [3-6]. Recently, for example, Laval and Daganzo proposed an effective implementation of the one-dimensional Euler's equation for lane-changing study [7].

\footnotetext{
Received Dec. 23, 2010; revision accepted Jan. 14, 2011

${ }^{*}$ Corresponding author. Tel.: +1-361-593-2270

Email: kfds000@tamuk.edu (D.Z. SUN)

doi: 10.3969/j.issn.2095-087X.2011.01.009
}

Computational fluid dynamics (CFD) began through investigation by Harlow in 1956 focusing on the movement of fluid materials under high compression [8]. In 1981, the first general purpose CFD package, PHOENICS, was developed and released by Concentration Heat and Momentum Limited (CHAM) [9]. However, as an important numerical method, CFD has not yet been implemented to solve the traffic flow problems. So far, only an implementation in determining piping in the transportation field has been reported [10]. This paper will focus on examining the application of CFD method to traffic flow analysis.

For Euler's equation implementations in transportation engineering, there are traditionally two viewpoints. The first is referred to as the Lagrangian description. This method concentrates on individual particles in a fluid flow, or individual vehicles in traffic study. Lagrangian description has been applied for studying certain traffic flow problems, such as car-following studies. When utilizing the Eulerian viewpoint, instead of individual vehicles in a flow, traffic is viewed as a simple continuously distributed flow, with consistent gaps between the cars constituting various levels of density, with more emphasis on given road segments. The methodology presented in this paper is based on the Eulerian description, and thus emphasis was placed on the flow as a whole or a system and not on the individual vehicles. 


\section{Methodology}

DOI: 10.1007/BF03325733

This section will describe the fundamental methodological steps related to the CFD approach. First the basic principles of CFD will be discussed followed by trafficspecific implementation issues.

\subsection{Fundamentals of CFD}

First, the one-dimensional Euler's equation will be deduced and explored. Let $C$ be a control volume $(C$ does not change with time, Fig.1 (a)). Due to the conservation of mass, the rate of change of mass in $C$ is

$$
\frac{\mathrm{d}}{\mathrm{d} t} m(C, t)=\frac{\mathrm{d}}{\mathrm{d} t} \int_{C} \frac{\partial k}{\partial t}(x, t) \mathrm{d} V=\int \frac{\partial k}{\partial t}(x, t) \mathrm{d} V,
$$

where $m$ is mass; $k$ is density.

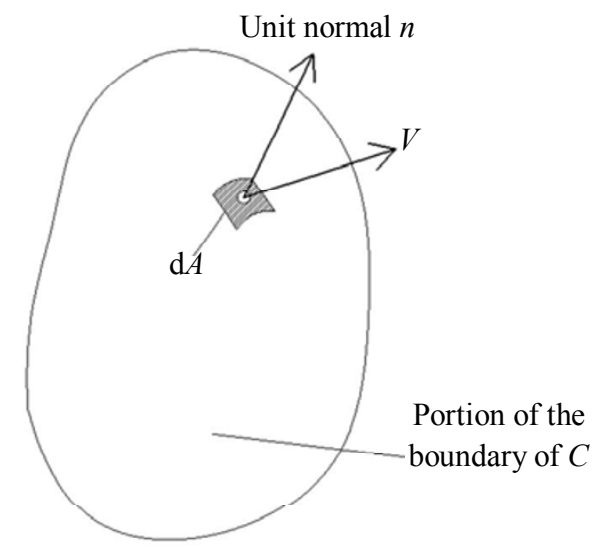

(a)

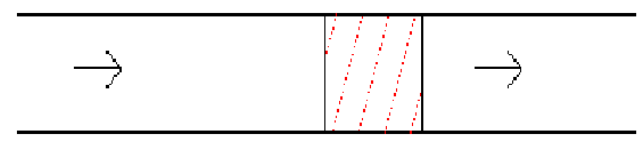

(b)

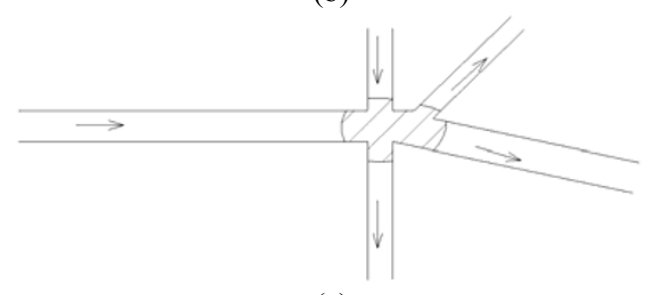

(c)

Fig. 1 The Eulerian description

The mass crossing the boundary $C$ per unit time equals the surface integral of $k \boldsymbol{v} \cdot \boldsymbol{n}$ over $\partial C$. The principle of conservation of mass can be more precisely stated as:

$$
\frac{\mathrm{d}}{\mathrm{d} t} \int_{C} k(x, t) \mathrm{d} V=-\int_{\partial C} k v \cdot n \mathrm{~d} A .
$$

Because this is true for all $C$, it is therefore equivalent to

$$
\frac{\partial k}{\partial t}+\operatorname{div}(k v)=0
$$

In traffic flow, $m$ is the number of vehicles on a road. The generalized equation (4) works not only in onedimensional linear roadway segment in most situations (Fig. 1(b)), but also in two-dimensional problems (Fig. 1(c)).

The generalized equation (Eq. (4)) is simplified to the 1D condition:

$$
\frac{\partial k}{\partial t}+\frac{\partial k \cdot v}{\partial x}=0 .
$$

To implement the CFD approach, one necessary assumption is that there exists some empirical relationship between speed and density, that is, the relationship between the flow $q$ and the density (concentration) $k$. This relationship between $q$ and $k$ might vary with location $x$ but not with time $t$, i.e.,

$$
q(x, t)=q^{*}(k(x, t)) .
$$

For some given function $q^{*}$, the conservation equation develops into:

$$
\frac{\partial k(x, t)}{\partial t}+\frac{\partial q^{*}(k(x, t))}{\partial x}=0 .
$$

This equation now only has two independent factors, location $x$ and time $t$.

When discussing the problem of a shock wave, Newell [5] emphasized the meaning of the relationship between the two independent factors. If the location $x^{*}$ is given, the slope of $q\left(x^{*}, t\right)$ can be found. And when the time $t^{*}$ is given, the slope of $k\left(x, t^{*}\right)$ can be found. The discontinuity of the slope represents the shock wave. So it is not necessary to track the actual path of the shock wave to determine the time at which a shock passes a given location, or the location which a shock arrives at a given time.

Typically, it is difficult to obtain a perfect mathematical solution to partial differential equations. Therefore, numerical solution methods have been widely used; however, the initial and boundary conditions need to be specified beforehand. The foundation of numerical methods is the Taylor formula:

$$
\begin{gathered}
k_{i}^{n+1}=k_{i}^{n}+\Delta \frac{\partial k}{\partial t}+\frac{\Delta t^{2}}{2} \frac{\partial^{2} k}{\partial t^{2}}+ \\
\frac{\Delta t^{3}}{6} \frac{\partial^{3} k}{\partial t^{3}}+\frac{\Delta t^{4}}{24} \frac{\partial^{4} k}{\partial t^{4}}+o\left(\Delta t^{5}\right), \\
k_{i}^{n-1}=k_{i}^{n}-\Delta \frac{\partial k}{\partial t}+\frac{\Delta t^{2}}{2} \frac{\partial^{2} k}{\partial t^{2}}- \\
\frac{\Delta t^{3}}{6} \frac{\partial^{3} k}{\partial t^{3}}+\frac{\Delta t^{4}}{24} \frac{\partial^{4} k}{\partial t^{4}}+o\left(\Delta t^{5}\right),
\end{gathered}
$$




$$
\begin{aligned}
& \begin{array}{c}
q^{n}{ }_{i+1}=q_{i}^{n}+\Delta x \frac{\partial q}{\partial x}+\frac{\Delta x^{2}}{2} \frac{\partial^{2} q}{\partial x^{2}}+ \\
\text { DOI: } 10.1007 / \mathrm{BF} 03325733
\end{array} \\
& \frac{\Delta x^{3}}{6} \frac{\partial^{3} q}{\partial x^{3}}+\frac{\Delta x^{4}}{24} \frac{\partial^{4} q}{\partial x^{4}}+o\left(\Delta x^{5}\right), \\
& q^{n}{ }_{i-1}=q_{i}^{n}-\Delta x \frac{\partial q}{\partial x}+\frac{\Delta x^{2}}{2} \frac{\partial^{2} q}{\partial x^{2}}- \\
& \frac{\Delta x^{3}}{6} \frac{\partial^{3} q}{\partial x^{3}}+\frac{\Delta x^{4}}{24} \frac{\partial^{4} q}{\partial x^{4}}+o\left(\Delta x^{5}\right),
\end{aligned}
$$

where $o$ denotes the error; $q_{i}^{n}$ and $k_{i}^{n}$ are the traffic volume and the density when $x=i \Delta x$ and $t=n \Delta t$.

In this step, although location $x$ and time $t$ are step functions, if $\Delta x$ and $\Delta t$ are small enough, and the results deduced are accurate enough for the transportation problems, the location $x$ and time $t$ can be still treated as continuous. Taylor formula can be changed to a difference format. For forward difference,

$$
\begin{aligned}
& \frac{\partial k}{\partial t}=\frac{k_{i}^{n+1}-k_{i}^{n}}{\Delta t}-\frac{\Delta t}{2} \frac{\partial^{2} k}{\partial t^{2}}- \\
& \frac{\Delta t^{2}}{6} \frac{\partial^{3} k}{\partial t^{3}}-\frac{\Delta t^{3}}{24} \frac{\partial^{4} k}{\partial t^{4}}+o\left(\Delta t^{5}\right) . \\
& \frac{\partial q}{\partial x}=\frac{q^{n}{ }_{i+1}-q_{i}^{n}}{\Delta x}-\frac{\Delta x}{2} \frac{\partial^{2} q}{\partial x^{2}}- \\
& \frac{\Delta x^{2}}{6} \frac{\partial^{3} q}{\partial x^{3}}-\frac{\Delta x^{3}}{24} \frac{\partial^{4} q}{\partial x^{4}}+o\left(\Delta x^{5}\right) .
\end{aligned}
$$

For backward difference,

$$
\begin{aligned}
& \frac{\partial k}{\partial t}=\frac{k_{i}^{n}-k_{i}^{n-1}}{\Delta t}-\frac{\Delta t^{2}}{2} \frac{\partial^{2} k}{\partial t^{2}}+ \\
& \frac{\Delta t^{3}}{6} \frac{\partial^{3} k}{\partial t^{3}}-\frac{\Delta t^{4}}{24} \frac{\partial^{4} k}{\partial t^{4}}+o\left(\Delta t^{5}\right), \\
& \frac{\partial q}{\partial x}=\frac{q_{i}^{n}-q^{n}{ }_{i+1}}{\Delta x}-\frac{\Delta x^{2}}{2} \frac{\partial^{2} q}{\partial x^{2}}+ \\
& \frac{\Delta x^{3}}{6} \frac{\partial^{3} q}{\partial x^{3}}+\frac{\Delta x^{4}}{24} \frac{\partial^{4} q}{\partial x^{4}}+o\left(\Delta x^{5}\right) .
\end{aligned}
$$

Using the difference formats of the Taylor formula, Eq. (7) is changed into the following format:

The difference of $k+$ the difference of $q=0$,

where the items in the differences with high order small amount $\Delta t$ or $\Delta x$ will be ignored.

To maintain sufficient accuracy, forward difference is applied first, followed by backward difference (which is at times referred to as MacCormack's method):

$$
\left.\begin{array}{l}
k_{i}^{\overline{n+1}}=k_{i}^{n}-\frac{\Delta t}{\Delta x}\left(q_{i}^{n}-q_{i-1}^{n}\right), \\
k_{i}^{\overline{\overline{n+1}}}=k_{i}^{\overline{n+1}}-\frac{\Delta t}{\Delta x}\left(q_{i+1}^{\overline{n+1}}-q_{i}^{\overline{n+1}}\right), \\
k_{i}^{n+1}=\frac{1}{2}\left(k_{i}^{n}+k_{i}^{\overline{n+1}}\right) .
\end{array}\right\}
$$

As described in (6), if there exists some relationship $q^{*}$ between $q$ and $k$, computational algorithms can be employed to calculate the parameters for a given road. To keep solutions stable, the following constraint condition is required:

$$
\left|k_{\max }\right| \frac{\Delta t}{\Delta x} \leq 1
$$

\subsection{Implementation methodology of CFD in traffic flow}

For numerical computation the basic concepts described previously can be deployed via the following simple steps. First, the initial condition must be given, which is the initial density. The iterations can then be started. Finally, conditions are applied to terminate the iterative procedure. The process can generate various outputs depending on the research requirements.

\subsubsection{Study the path of shock waves}

If we want to know at a given time $t^{*}=n \Delta t$ where the shock wave is, we can adopt the stop condition, and output the densities of any location $x_{i}$. The location of the discontinuity of the density is the location of the shock wave.

On the other hand, if we want to know at a given location $x^{*}=j \Delta x$ when the shock wave will arrive, we can adopt the stop condition:

$$
x_{j}-x_{j-1}>\delta \text {. }
$$

In practice, the programmer usually adopts $x_{j-a}$ ( $a$ is a small integer). Consider the shakes in the location of the discontinuous point, and output the number of iterations $n . n \Delta t$ is the time that the shock wave reaches the given location.

\subsubsection{Estimate traffic volume}

The traffic volume is easily calculated by the given function $q^{*}$ after acquiring the density in any location. We can use the volumes of the input and output of a region to calculate the number of vehicles within the region. Continuously monitoring this parameter can help identify whether there is a breakdown in this region, even if no data about density or volume is collected within this region [11]. First, the initial condition is needed. The initial number of vehicles $N_{0}$ can be calculated as:

$$
N_{0}=\sum_{i=j}^{j+e} k_{i}^{0} \Delta x,
$$

where $k_{j}^{0}$ is the density in the input of the region; $k_{j+e}^{0}$ is the density in the output of the region; $e \Delta x$ is the length of the region on the road. There are two methods to cal- 
culate the time $n \Delta t$ and the number of vehicles $N_{n}$. One employs the density, which is similar to (20):

DOI: $10.1007 / \mathrm{BF} 03325733$

$$
N_{n}=\sum_{i=j}^{j+e} k_{i}^{n} \Delta x,
$$

$e k_{i}^{n}$ is the density at the time $n \Delta t$.

The other, employs the in and out volumes of a given region. During the time $n \Delta t$, the number of vehicles that traveled into and out of the region are:

$$
\begin{aligned}
& N_{\text {in }}=\sum_{0}^{n} q_{j}^{n} \Delta t, \\
& N_{\text {out }}=\sum_{0}^{n} q_{j+e}^{n} \Delta t .
\end{aligned}
$$

Therefore, at the time $n \Delta t$, the number of vehicles $N_{n}$ is:

$$
\begin{aligned}
& N_{n}=N_{0}+N_{\text {in }}-N_{\text {out }}= \\
& \sum_{i=j}^{j+e} k_{i}^{0} \Delta x+\sum_{0}^{n} q_{j}^{n} \Delta t-\sum_{0}^{n} q_{j+e}^{n} \Delta t .
\end{aligned}
$$

The computational workload depends on the length of time and the length of the region. To reduce the computational workload, method 1 is preferred for longer time periods; otherwise method 2 is better for longer road regions.

\subsubsection{Study the time-space diagram}

The time-space diagram has been widely used for solving some traffic-related problems such as gap studies. In some time $t_{0}$, a vehicle is in the location $S_{0}$ where the density is $k_{1}$. Then, the velocity $v_{1}$ in this location can be computed through the relationship between $q$ and $k$ ( $\operatorname{since} q=k v$ ). Next, the distance traveled by the vehicle during the first time segment $\Delta t$ is calculated with the equation

$v_{1} \Delta t$ (for the first iteration of time),

and the location in this time is

$$
S_{1}=S_{0}+v_{1} \Delta t .
$$

In the next time segment $t_{m}+\Delta t$, the density $k_{2}$ is determined in the location $S_{1}$, and then the velocity $v_{2}$ is computed in this location through the relationship between $q$ and $k$. Therefore, the distance traveled by the vehicle during the second time segment $\Delta t$ and the location can be calculated:

$v_{2} \Delta t$ (for the second iteration of time),

$S_{2}=S_{1}+v_{2} \Delta t$.

...

By analogy, after $m$ segments of time $\Delta t$,

$S_{m}=S_{m-1}+v_{m} \Delta t$.
From the relationship between the time and the distance, the T-S diagram can then be drawn.

\subsubsection{A sample implementation}

In the following examples, a simplified relationship between the velocity $v$ and the density $k$ is adopted:

$$
\begin{aligned}
& v=-2.5 k+100, \\
& q=-2.5 k^{2}+100 k .
\end{aligned}
$$

Problem description:

On a road which is 10 miles long, there exist two one mile long queues in the location $x=2$ mile and $x=6$ mile, respectively (Fig. 2). Note, the locations with elevated density represent queues. In the first part of the road, the lower density is 15 vehicles per mile (vpm), and the higher density is $30 \mathrm{vpm}$. The volume is the maximum at the location seven miles away from the starting point, where the density is

$$
k=\frac{k_{\max }}{2}=20 \mathrm{vpm} \text {. }
$$

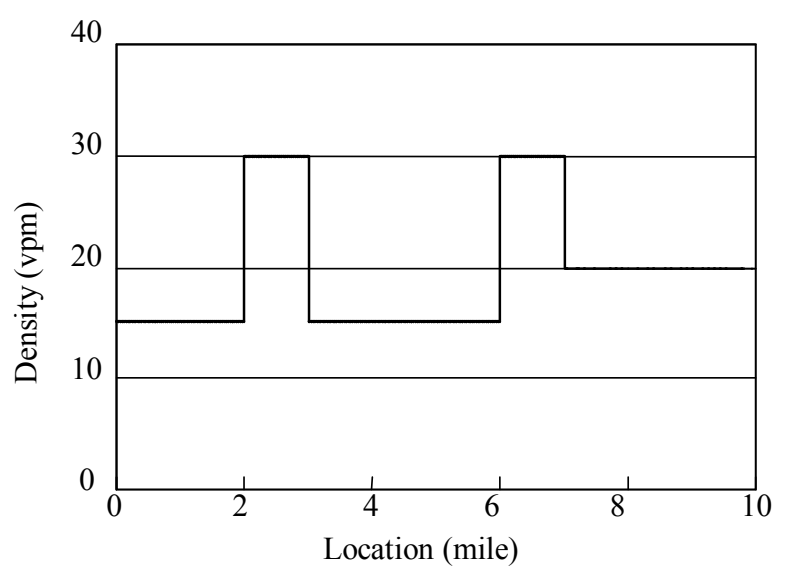

Fig. 2 The initial condition of the example

Problems:

(1) Present the movement of traffic flow waves.

(2) Detect the number of vehicles in a 2-mile long region from $x=3$ mile to $x=5$ mile.

(3) Plot the trajectories of two selected vehicles at the location $x=0$ mile and $x=2$ mile.

Solution:

From (18),

$$
\left|k_{\max }\right| \frac{\Delta t}{\Delta x} \leq 1,\left|k_{\max }\right|=40 \mathrm{vpm} .
$$

Choose $t=0.0001, \Delta x=0.01$ and then

$$
\left|k_{\max }\right| \frac{\Delta t}{\Delta x}=40 \frac{0.0001}{0.01}=0.4 \leq 1,
$$

which implies that the road is divided into $1000 \mathrm{sec}-$ tions, and the accuracy of time is $0.0001 \mathrm{~h}$.

(1) For the termination condition of the iterative procedure, this research adopts that when $k_{i}^{n+1}-k_{i}^{n}<0.01$, 
stop the iteration ( $i$ is the space coordinate, which represents a random point in the space; $n$ is the time coordi-

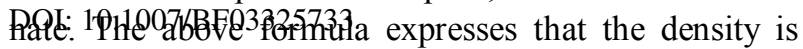
kept stable after a segment of time $\Delta t$ from $n \Delta t$ to). The time of the iteration is $n=4113$, which means after $0.4113 \mathrm{~h}$, the traffic flow becomes stable in the 10 mile region.

Fig. 3 shows the density-location diagram for every $0.07 \mathrm{~h}$ (every 700 iterations).

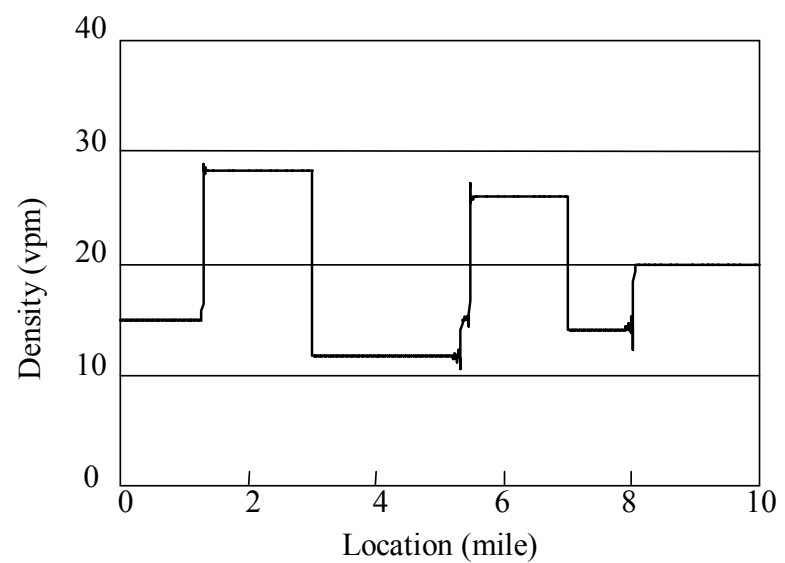

(a) $0.07 \mathrm{~h}$

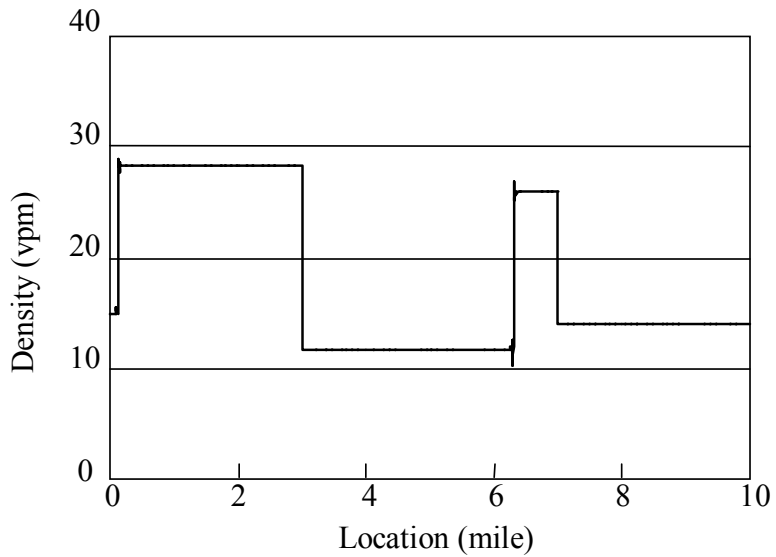

(c) $0.21 \mathrm{~h}$

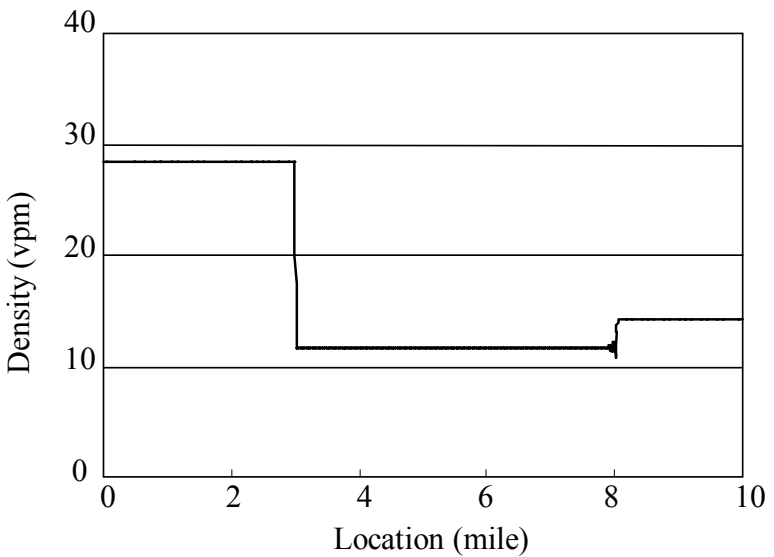

(e) $0.35 \mathrm{~h}$
(2) In each iteration, we calculate the number of vehicles in the region from $x=3$ to $x=5$, by (17):

$$
N_{n}=\sum_{i=300}^{500} k_{i}^{n}(0.01) \text {. }
$$

Then, we can draw the curve according to the change in the number of vehicles in the given region, as shown in Fig. 4.

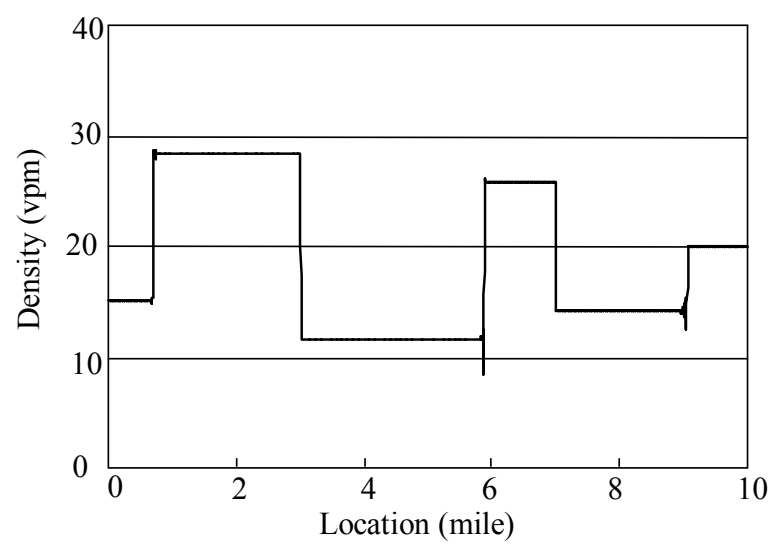

(b) $0.14 \mathrm{~h}$

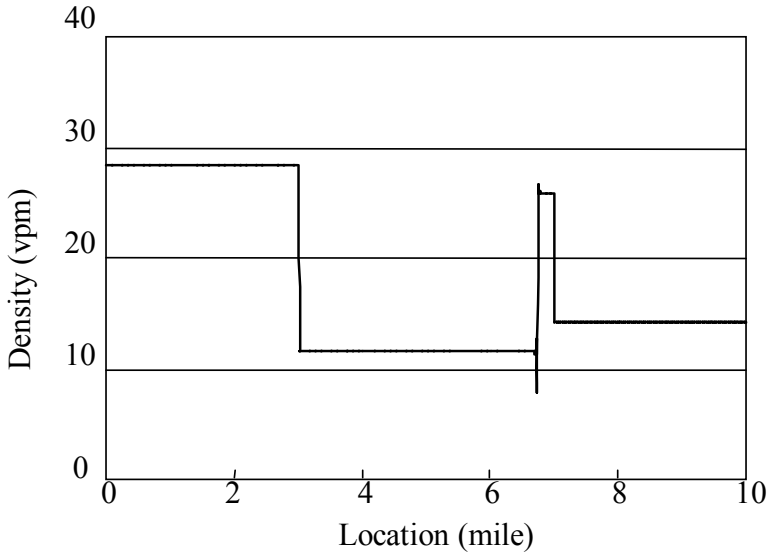

(d) $0.28 \mathrm{~h}$

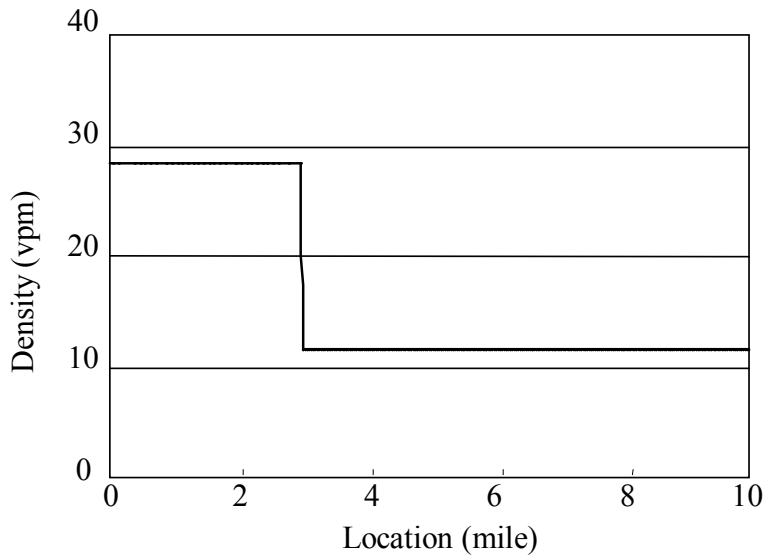

(f) $0.42 \mathrm{~h}$

Fig. 3 The time-space diagrams for different vehicles 


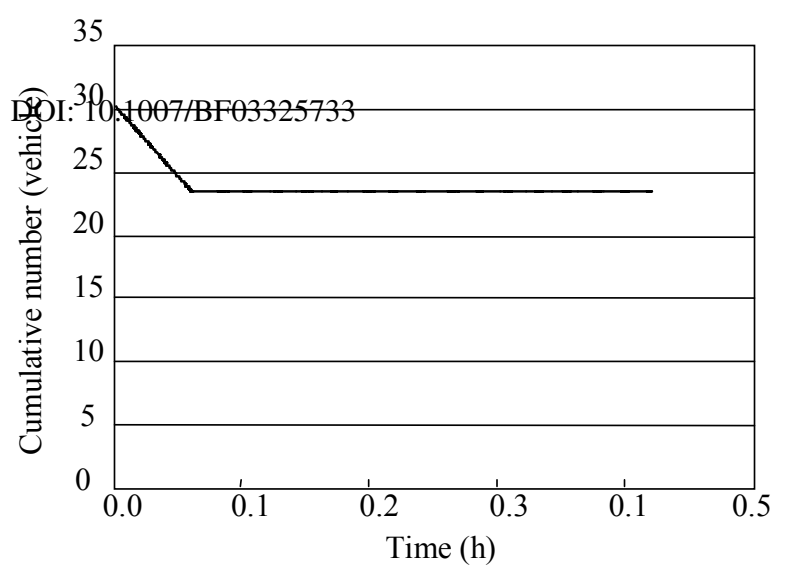

Fig. 4 The number of vehicles on a given road segment

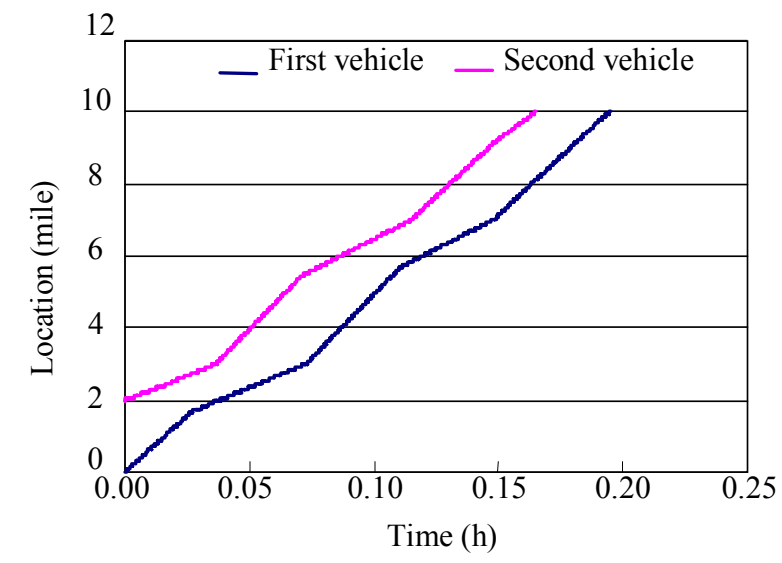

Fig. 5 The Time-space diagrams for different vehicles

(3) For the first vehicle at the location $x=0$ mile, give the initial condition $S_{0}=0$ mile. Because $\Delta x=0.01$, when we get $S_{m}(m=1,2,3, \cdots)$, which should be rounded off to two decimal places in the computer program, Eq. (22) is developed into the following format:

$$
S_{m}=S_{m-1}+v_{\operatorname{lnt}\left(100_{\mathrm{m}-1}\right)} \Delta t .
$$

For the second vehicle at the location $x=2$ mile, the initial condition $S_{0}=2$ mile is given. Because of the movements of the waves, the space between the two vehicles varies, as described in Fig. 5.

\section{Some theoretical and practical applica- tions of CFD in transportation}

The CFD method could be applied to solve both theoretical and practical problems in the transportation area for several reasons. First, because the CFD method can clearly describe the propagations of waves of traffic flows, it is able to explain or even identify some surprising phenomena in traffic flow theory; secondly, not only can the CFD method estimate the traffic conditions in the downstream as well as the future, but also in the upstream and in the past, which will be very helpful to maximize the utilization of limited existing detector systems. The CFD method can help reduce the requirement of detector numbers and even supplement the data when some detectors fail in a system.

\subsection{Some theoretical applications}

This section will describe two theoretical applications of the CFD approach. First the effect of the distribution of queues on congestion propagation will be discussed followed by the minimum gap between adjacent platoons.

\subsubsection{The effect of the distribution of queues (high- density platoons)}

It is well known that queue distribution, which can also be considered as high-density platoons, will impact system performance. When the two individual queues mentioned in Section 2.2.4 are replaced with one 2-mile single queue at the location $x=5$ mile (Fig. 6(a)), the traffic system performance will be changed, even though the total queue length is the same.

First, the process for the traffic flow with one queue to become stable requires more time (19300 iterations taking $1.9300 \mathrm{~h}$ ) than that with two queues (4 113 iterations taking $0.4113 \mathrm{~h}$ ). Second, the flow volumes in the outputs of the two are different. After the traffic flows are stable (every parameter in the given road is constant as time passes), the densities in the road of the two situations are also different (Fig. 6(c)).

Fig. 6(b) shows that the throughput of the one-queue situation is higher by approximately $10 \%$ than that with two queues. However, travelers are concerned more with traveling time than road throughput. When examining the density after the traffic flow is stable (Fig. 6(c), which is at the location after about $x=3$ mile), the density of the situation with one-queue is higher than that of situation with two queues. This indicates the velocity is slower when the vehicle is traveling through this segment of the road. The computation results also show that the situation with two short queues can save the total traveling time by about $20 \%$ compared with that with one long queue. This finding can be further applied to congestion management strategies.

\subsubsection{The control of the gap between two platoons}

The interaction between adjacent platoons is essential for the formation and propagation of congestions. In this section, the minimum gap between platoons is studied to help prevent congestion propagation. For example, in a given road segment, take a platoon with the density of $k_{0}$ vehicles per mile and the length of $a$ miles. It is followed by another platoon with the gap of $G$ miles be- 
tween the two (Fig. 7(a)). The leading vehicles of the two platoons had the same initial speed $v_{0}=\operatorname{function}\left(k_{0}\right)$

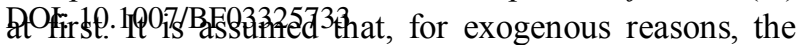
leading vehicle of the first platoon slows down for time $t_{0}$ and then recovers to the initial speed. Now, we want to discern the minimum gap $G$ so that the second platoon could avoid the impact of the speed reduction of the first platoon (i.e., the congestion will not be propagated into the second platoon).

In fact, the gap $G$ depends on $t_{0}$ : the longer $t_{0}$, the greater $G$. To avoid the impact of the first platoon on the

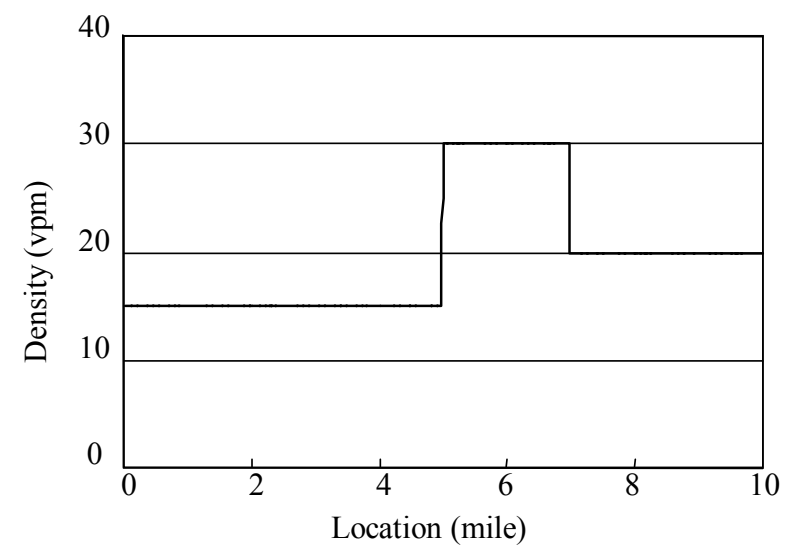

(a)

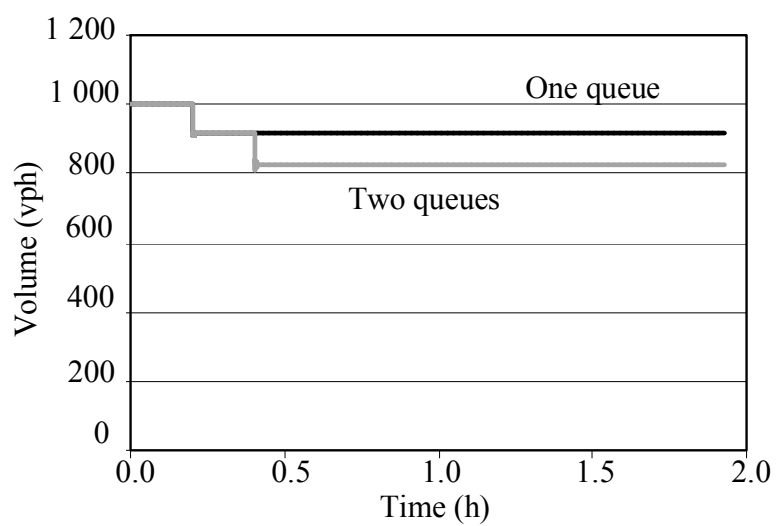

(b)

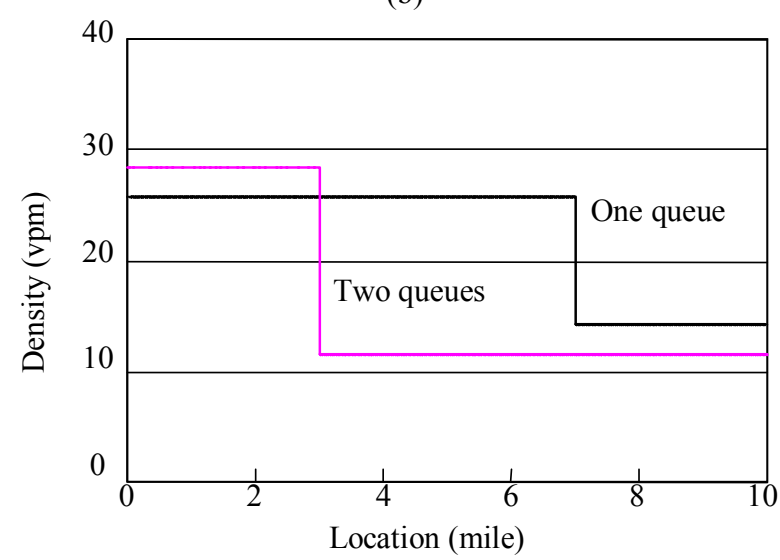

(c)

Fig. 6 The effect of the distribution of queues

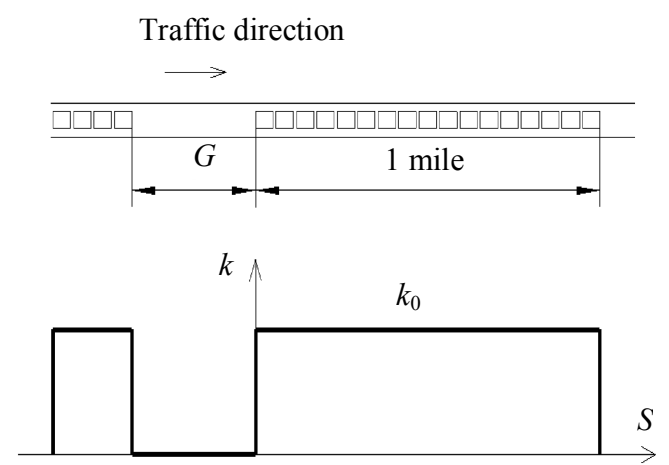

(a)

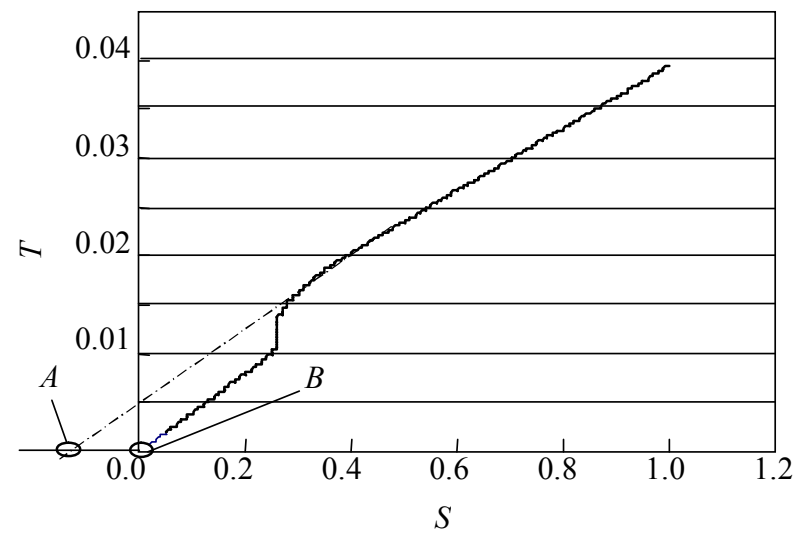

(b)

Fig. 7 The gap between two platoons

second, it necessitates that the trajectories of the last vehicle of the first platoon and the leading vehicle of the second platoon do not intersect. Fig. 7(b) shows the T-S (time-space) diagram of the first platoon when choosing $a=1$ mile, $k_{0}=30 \mathrm{v} / \mathrm{m}, t_{0}=0.005 \mathrm{~h} \mathrm{(18} \mathrm{s),} \mathrm{and} \mathrm{using} \mathrm{(27)}$ and (28) to describe the relationships of the speed, density and flow rate. In Fig. 7(b), point $A$ is the intersection of the tangent (slope $1 / v_{0}$ ) of the trajectory curve of the last vehicle in the first platoon with Space axis. Point $B$ is the intersection of the trajectory curve of the last vehicle in the first platoon with Space axis. So the distance between points $A$ and $B$ denotes the minimum gap required. The coordinate of point $A$ represents the location of the leading vehicle in the second platoon. According to Fig. 7(b), the gap $G$ should be at least 0.112 miles.

\subsection{Practical application}

A practical application of CFD is demonstrated with the real data from the Transguide [12] ITS program in San Antonio, Texas. The studied region is located on Interstate highway 10. Fig. 8(a) shows the distribution of 7 loop detectors on the studied road. The loop detectors collected the data about speed, volume and occupancy every 20 seconds. In this study, data from Sep- 
tember 15th, 2006 was randomly selected for analysis. Considering the distribution of detectors (Fig. 8(b)), PRI to the CFD method. The locations of detectors 4 and 7 were used to compare the predicted volume obtained via the CFD method versus the actual detector counts at these locations.

First, the empirical flow-density relationship using the data from detector 5 was plotted in Fig. 9(a); and then the predicted volumes were compared with the real observed values for detectors 4 and 7, shown in Fig. 9(b) and Fig. 9(c), respectively. It is found that the prediction errors for both detectors were small, while the result for detector 4 (average prediction error $=0.5 \%$ ) was obviously more accurate than that for detector 7 (average prediction error $=4.85 \%$ ). From the one-dimensional Euler's equation (5) and its assumptions, we know that the accuracy of the CFD method mainly depends on two factors: the reality of the speed-density-flow relationship and the external interruption to the traffic flow within the studied road segment. As the distance increases, it is hard to keep identical speed-flow-density relationship and exclude some external interruption to the traffic flow; therefore the predicted results of detector 4 was more accurate than that of detector 7 , which is farther from the base detector 5 .

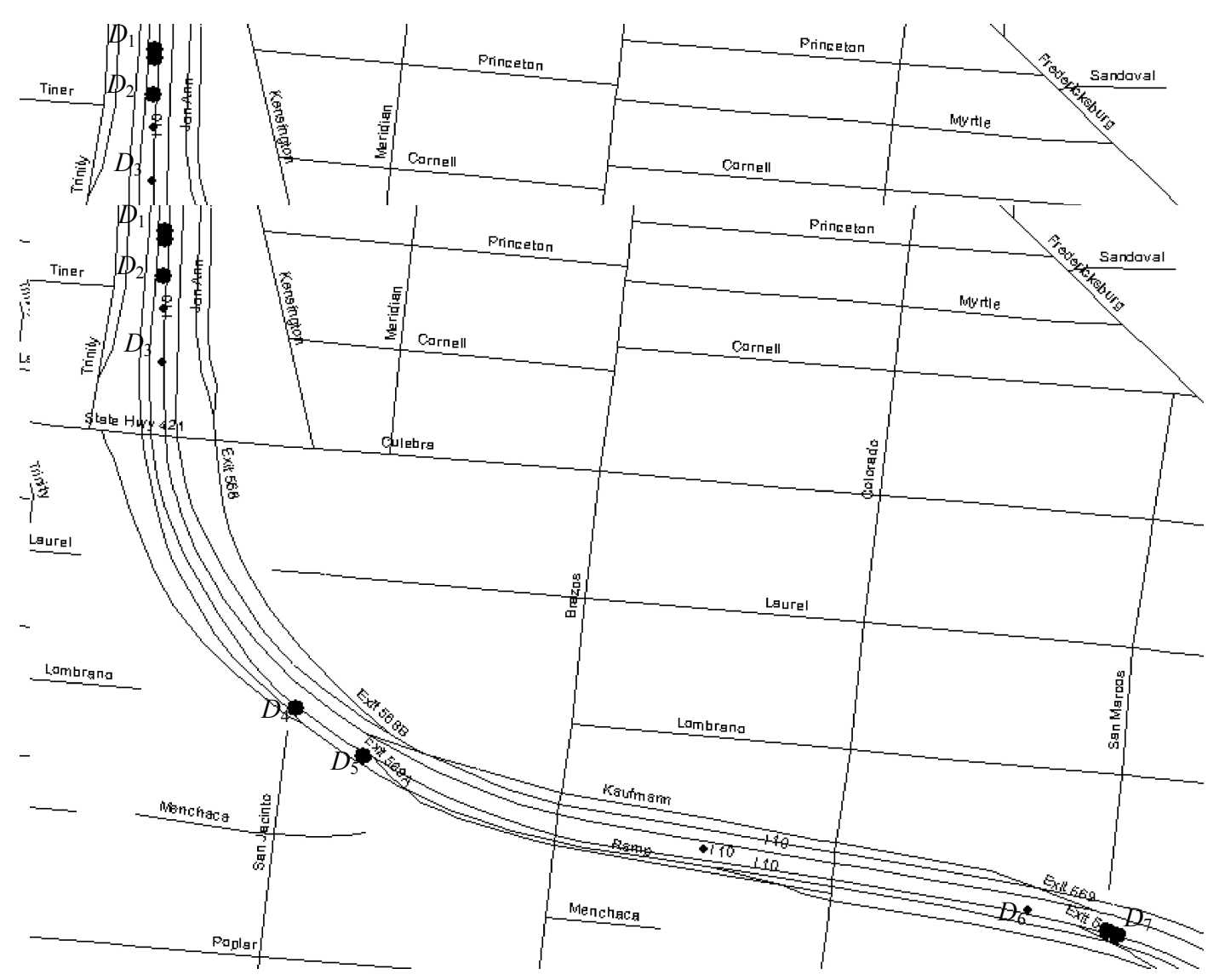

(a)

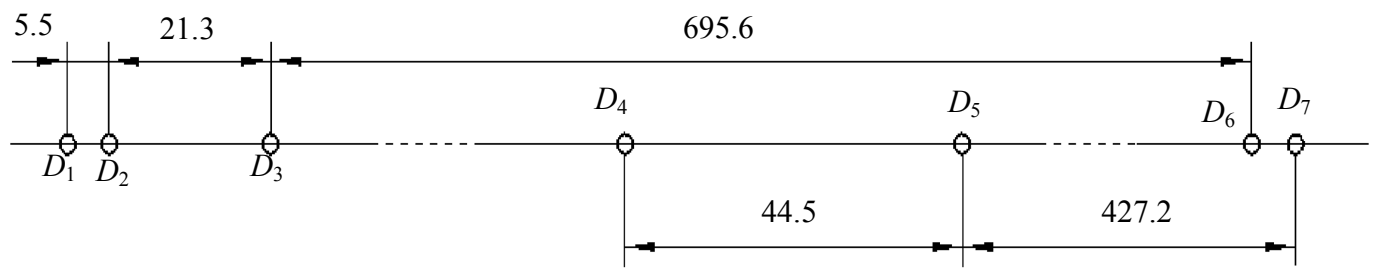

(b)

$D_{i}$ denotes the loop detectors; among them, $D_{1}, D_{2}, D_{3}$, and $D_{6}$ were set up on the west bound; $D_{4}, D_{5}$, and $D_{7}$ were set up on the east bound. The unit of the length is $10^{-3}$ mile.

Fig. 8 The distribution of the detectors 
Not only could the CFD method predict the downstream and future traffic conditions as the traditional

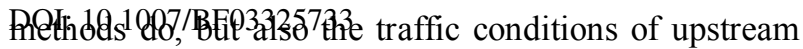
as well as the past time. It was observed that when estimating the upstream traffic conditions, the accuracy of the CFD method did not decrease (Fig. 9(b)). In addition, when inversing the coordinates of the discrete points in the studied road segment, it can even estimate traffic conditions in the past.

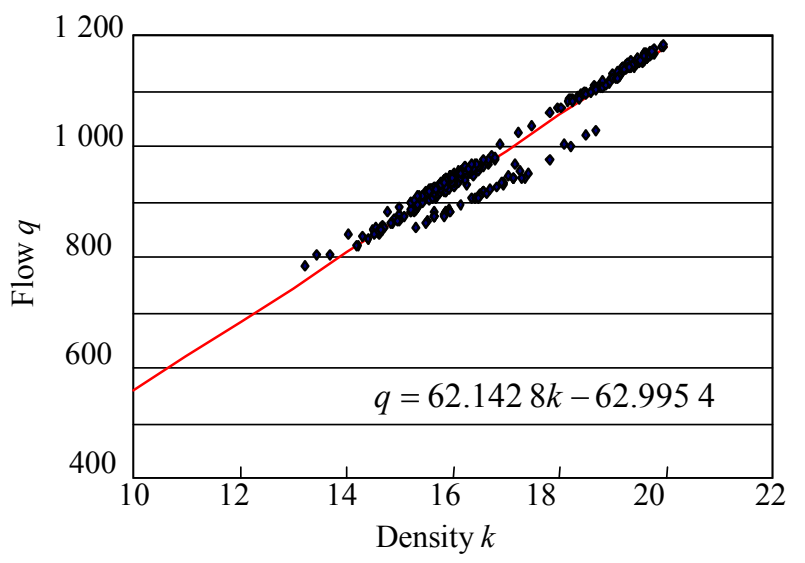

(a) Flow-density relationships

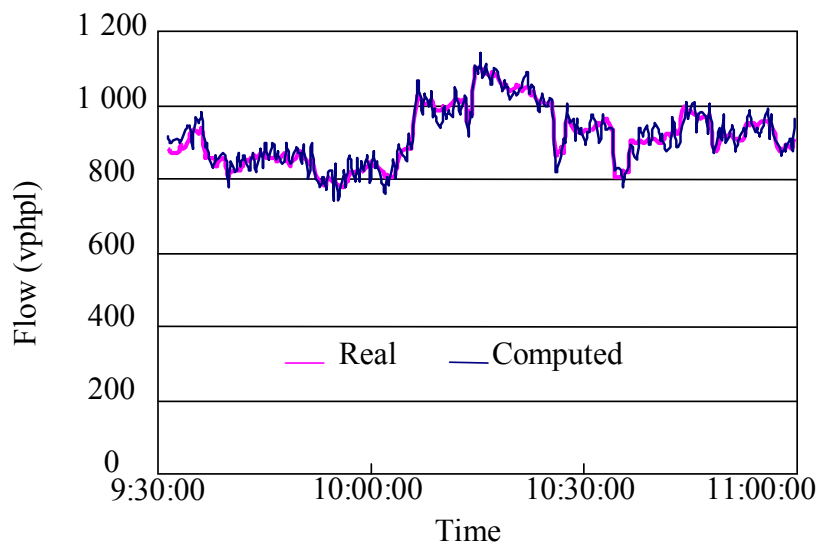

(b) The flow rate measured by $D_{4}$

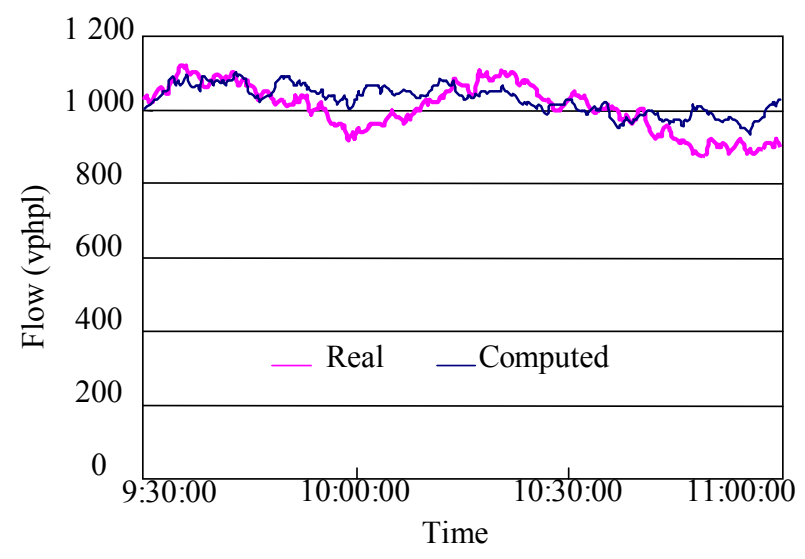

(c) The flow rate measured by $D_{7}$

Fig. 9 The validation by using real data from Transguide
Fig. 10 illustrates the development of the traffic flow. The cluster of lines $\boldsymbol{L}$ on the Time-Space plane represents for the trajectories of each vehicle. As time passes, a rectangular region will be formed over the road segment with four boundaries. The boundaries $\boldsymbol{A}$ and $\boldsymbol{B}$ represent the traffic conditions, in terms of density, in the past and in the future, respectively. The boundaries $\boldsymbol{C}$ and $\boldsymbol{D}$ represent the traffic conditions, in terms of flow rate, in the upstream and in the downstream, respectively. Given any one boundary, the other three can be calculated. This advantage of the CFD method could also be used to enhance the reliability of the detector system. When certain detectors can not work properly, the CFD method could supplement the data for these non-functioning detectors. Moreover, if the density or flow rate discontinuities are derived from the two sides of the boundary rectangle $\boldsymbol{A}$ and $\boldsymbol{B}$ or $\boldsymbol{C}$ and $\boldsymbol{D}$, it may indicate that incidents or congestion occurred on the studied road segment.

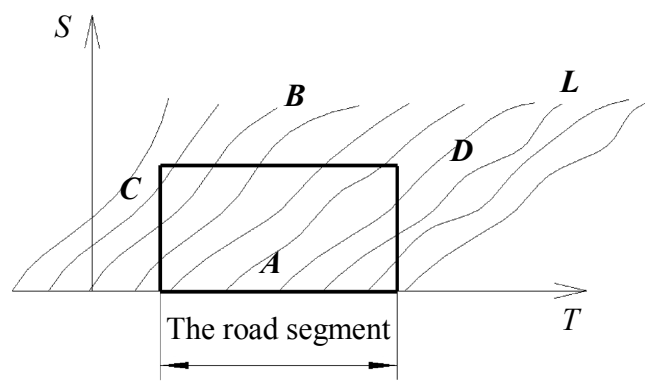

Fig. 10 The development of the traffic flow

\section{Conclusions}

This paper first introduced the fundamentals of the CFD approach along with simple implementation examples in for traffic analysis. As a numerical solution, the CFD method has three advantages compared with general traffic simulation: (1) it can reduce the computational workload; (2) it can provide more detailed description regarding traffic flow waves; (3) it can estimate traffic conditions of the upstream as well as the past. However, the accuracy of the CFD method is bound to two limited assumptions. First, there exists a speed-density-flow relationship; second, the studied road segment has minimum external interruptions. The applicability and capabilities of the method were demonstrated through theoretical analysis as well as field data collected in San Antonio, Texas.

The theoretical and practical applications discussed in this paper revealed the great potential of the CFD approach for transportation study. In traffic theory analysis, CFD can be applied to investigate the impact of queue/platoon distribution on congestion formation and 
propagation. In practice, the CFD method can be utilized to predict the traffic condition of a system based on

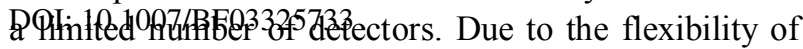
the CFD method for estimating the traffic conditions in the downstream in the future, but also in the upstream and in the past, CFD can be utilized to enhance the reliability of the detector system. Moreover, CFD can be used to process the data from a limited number of traffic detectors, therefore to predict the location and time of congestion (or incidents) for certain road segments without existing detectors.

\section{References}

[1] M.J. Lighthill, G.B. Whitham, On kinematic waves. I: flood movement in long rivers. II: a theory of traffic flow on long crowded roads, In: Proceedings Royal Society, Series A, Mathematical and Physical Sciences, London, 1955: 281-316.

[2] P.I. Richards, Shock waves on the highway, Operations Research, 1956, 4(1): 42-51.

[3] H.J. Payne, FREFLO: a macroscopic simulation model of freeway traffic, Transportation Research Record, 1979(722): 68-77.

[4] N.A. Derzko, A. J. Ugge, E. R. Case, Evaluation of dynamic freeway flow model by using field data, Transportation Research Record, 1983(905): 52-60.
[5] G.F. Newell, A simplified theory of kinematic waves in highway traffic, part I: general theory, Transportation Research-B, 1993, 27(4): 281-287.

[6] H.M. Zhang, Analyses of the stability and wave properties of a new continuum traffic theory, Transportation Research-B, 1999, 36(9): 399-415.

[7] C.F. Daganzo, In traffic flow, cellular automata $=$ kinematic waves, Transportation Research-B, 2006, 40(5): 396-403.

[8] L.J. Norman, The legacy and future of CFD at Los Alamos, In: Canadian '96 CFD Conference, http://www. lanl.gov/orgs/t/t3/secondlevel/history/viewgraphs.pdf, 1996-03-03.

[9] Professor Spalding's biography, http://www.ham.co. uk/website/new/dbs.htm, 2007-07-15.

[10] J.E. Berlamont; K. Trouw, G. Luychx, Shear stress distribution in partially filled pipes, Journal of Hydraulic Engineering, 2003, 129(9): 697-705.

[11] K. Chung, J. Rudjanakanoknad, M. J. Cassidy, Relation between traffic density and capacity drop at three freeway bottlenecks, Transportation Research-B, 2007, 41(1): 82-95.

[12] Transguide, http://www.transguide.dot.state.tx.us/lanedata/, 2007-07-15.

(Editor: Yao ZHOU) 